\title{
Nasopharyngeal Cancer: Prevalence, Outcome, and Impact on Health-Related Quality of Life at Princess Norah Oncology Center, Jeddah, Saudi Arabia
}

Nabil Alsafadi ${ }^{1}$, Mohammed S. Alqarni ${ }^{2}$, Meshari Attar ${ }^{2}$, Rayan Mgarry ${ }^{2}$, Abdulhameed Bokhari ${ }^{3}$

1. Radiation Oncology, King Abdulaziz Medical City, National Guard Hospital Jeddah, Jeddah, SAU 2. Medicine, King Saud Bin Abdulaziz University for Health Sciences, Jeddah, SAU 3. Urology, King Saud Bin Abdulaziz University for Health Sciences / International Medical Center, Jeddah, SAU

Corresponding author: Nabil Alsafadi, nabilalsafadi1954@gmail.com

\section{Abstract \\ Introduction}

Head and neck malignancies are considered among the most common cancers that arise from different anatomical sites in the region. The number of new cases diagnosed worldwide each year is estimated to be more than 550,000 resulting in about 380,000 deaths. One of these head and neck cancers that may affect patient quality of life is the nasopharyngeal carcinoma (NPC). The purpose of our study is to assess the outcome, and the quality of life of these patients. Our study reviews NPC patients treated at Princess Norah Oncology Center, Jeddah, retrospectively over the past 15 years to provide additional information on this disease in Saudi Arabia.

\section{Methods}

We included all histologically confirmed cases of NPC seen at National Guard Hospital in Jeddah between 2002 and 2017. The data was collected retrospectively from the BestCare system, hospital information system, and the medical records. The created table included demographics, comorbidities, and first symptoms. The research table also contained stage at presentation and treatment modalities. Moreover, 25 patients were asked to complete the Arabic versions of European Organisation for Research and Treatment of Cancer Quality of Life Questionnaire Head and Neck Module (EORTC HN-35) module questionnaire to assess the quality of life. All results were computed using IBM SPSS version 23 (IBM Corp., Armonk, NY), which was provided by College of Medicine at KSAU.

\section{Results}

Received 05/01/2020

Review began 05/09/2020 Review ended 05/11/2020 Published 05/19/2020

\section{(c) Copyright 2020}

Alsafadi et al. This is an open access article distributed under the terms of the Creative Commons Attribution License CC-BY 4.0., which permits unrestricted use, distribution, and reproduction in any medium, provided the original author and source are credited.
A total of 107 patients with adequate documentation were identified. There were $72.9 \%$ males and $27.1 \%$ females; $81.3 \%$ of patients were alive and in remission and $18.7 \%$ were dead. Neck mass was the most common clinical manifestation present in $84.1 \%$ of patients. Radiotherapy and chemotherapy were the most used modality by $96.3 \%$. The five-year survival rate year was $81.3 \%$. Moreover, the $\mathrm{H} \& \mathrm{~N}-35$ questionnaire showed that the NPC survivors suffered mostly poor social contact.

\section{Conclusion}

A large proportion of the identified patients were in remission. Quality of life assessment shows that the main impact of the disease and treatment was on social contact.

Categories: Radiation Oncology, Oncology

Keywords: nasopharyngeal carcinoma, outcomes, heath related quality of life, prevalence, kingdom of saudi arabia (ksa)

\section{Introduction}

According to the World Health Organization (WHO), cancer is one of the leading causes of mortality and morbidity globally [1]. Head and neck malignancies are among the most common cancers that arise from different anatomical sites in the region. The number of new cases diagnosed worldwide each year is estimated to be more than 550,000 cases resulting in about 380,000 deaths $[2,3]$.

A specific type of cancer that occurs between the head and neck region is nasopharyngeal cancer (NPC), which is very rare in the West [4]. Worldwide, the incidence of NPC is slightly less than one per 100,000 population annually [5]. In Saudi Arabia, NPC is seen in 6\% of all malignancies that are diagnosed yearly with an incidence of 0.8 per 100,000 [6]. NPC is the most common type of malignancy arising in the nasopharynx, the narrow tubular passage behind the nasal cavity $[7,8]$. It is characterized often by poorly or 
undifferentiated carcinoma. It differs from non-nasopharyngeal head and neck squamous cell carcinomas in several ways, including its association with the Epstein-Barr virus (EBV), increased radio-chemo sensitivity, and a greater propensity for distant metastases [9]. The risk to develop NPC seems related to several factors, such as EBV latent infection, environmental factors, which includes the high intake of preserved foods along with smoking, and genetic predisposition [10,11]. In NPC viral DNA infects epithelial cells and is associated with their transformation to cancer.

The genetic factor seems to impact the development of the NPC dramatically $[12,13]$. There is a high rate of this cancer within some specific ethnic groups. Patients that have A2 HLA haplotypes, and cytogenetic abnormalities identified within the tumor samples [14]. Clinical presentations related to NPC may not appear in early stages. However, in late stages, NPC includes apparent complications such as swollen lymph nodes around the neck, blood in the nose and saliva, hearing loss and ear infection $[14,15]$. Moreover, advanced NPC can lead to a multiple of neurological associated symptoms, including unilateral deafness and difficulty in opening the mouth, and this is known as Trotter's syndrome [16]. Serious complications of nasopharyngeal carcinoma can also occur during treatment. According to a review article by Suarez et al., radiotherapy and radio-surgery for NPC can include some complications such as nasopharyngeal necrosis, temporal lobe necrosis, cranial nerve palsies and cerebral edema mainly in patients retreated for local recurrence [17]. In addition to that nasopharyngeal cancer can adversely affect quality of life, including emotional, social and psychological abnormalities [18]. NPC patients can also be presented with difficulty swallowing, hearing loss, and speech difficulties [19]. Therefore, it is important to assess quality of life in these patients.

The purpose of our study is to find the morbidity, functional status and to assess the quality of life of these patients. Our study reviews NPC patients treated at Princess Norah Oncology Center, Jeddah, retrospectively over the past 15 years to provide additional information on nasopharyngeal cancer patients in Saudi Arabia.

\section{Materials And Methods}

The records of 141 clinical data were collected from all histologically confirmed cases of NPC which took place between 2002 and 2017, at National Guard Hospital in Jeddah, Kingdom of Saudi Arabia. The inclusion and exclusion criteria were retrospectively reviewed on hard files and soft files (BestCare). Patients that had been diagnosed before 2002 or had other nasopharyngeal malignancies were excluded. The research started by gathering 141 patients' files, and 34 NPC patients were excluded because their files were either not complete or were lost follow-up, so we included 107 NPC patients in the incidence of the study.

A table was created that included the following demographics: age, gender, remission status and death status. Headache, hearing loss, and vision disturbances were the symptoms included in the table. The research table also contained stage of cancer at presentation, tumor nodes metastasis (TNM) stages, radiation, chemotherapy, surgery, hypertension and diabetes. Staging of the disease was stated according to the Cancer Staging Manual by the American Joint Committee on Cancer, sixth edition [20]. All patients' information was viewed and then placed into the table accordingly. The treatment modalities were radiotherapy, chemotherapy and surgery.

Moreover, 25 patients, who attended the follow-up clinic during the study period and were free of the disease more than two years, were asked to complete Arabic versions of European Organisation for Research and Treatment of Cancer Quality of Life Questionnaire Head and Neck Module (EORTC HN-35) questionnaire to assess the quality of life related to the stage and treatment by the research coordinator. Sub-scales have measured pain, swallowing, sense, speech, social eating and contact, weight loss/gain, painkillers, ill feeling, coughing, feeding tube, open and dry mouth, teeth, sticky saliva and sexual problems. For the analysis, categorical variables were summarized by frequencies and percentages, and continuous variables by means and standard deviations, or medians and interquartile ranges if their distributions were skewed. Variables with $\mathrm{p}$ value less than .05 were considered significant. All results were computed using IBM SPSS version 23 (IBM Corp., Armonk, NY), which was provided by College of Medicine at KSAU.

\section{Results}

Our sample size included 107 patients. Patients in this study were ranged from 11 to 106 years with a mean age of 53.85 years $(\mathrm{SD}=15.72$ years), had a BMI ranged from 13.42 to 51.20 with a mean BMI of $(\mathrm{SD}=7.06)$ and the mean follow-up was 84.42 months ( $\mathrm{SD}=55.78)$. Among our population, $72.9 \%(\mathrm{n}=78)$ were males and $27.1 \%(\mathrm{n}=29)$ were females. They had co-morbidities such as diabetes $(25.2 \%, \mathrm{n}=27)$, hypertension $(29 \%, \mathrm{n}=31)$, and smoking $(9.3 \%, \mathrm{n}=10) ; 81.3 \%(\mathrm{n}=87)$ of our population presented with remission and $18.7 \%(\mathrm{n}=20)$ patients were dead. The patients' stages and clinical presentations are shown in Figure 1 and Tables 1,2 . 


\section{Cureus}

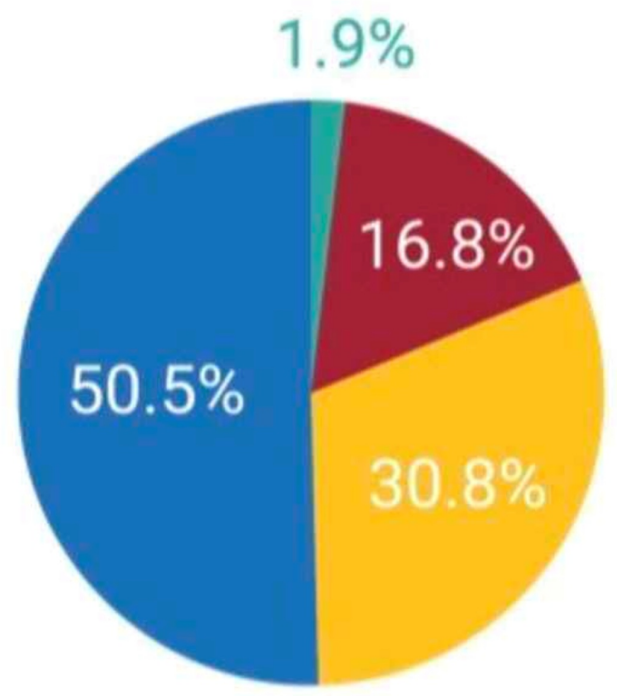

- Stage 1

- Stage 2

Stage 3

- Stage 4

FIGURE 1: Patients' stages

Clinical presentation

\section{Headache}

Nasal obstruction

Nasal bleeding

Visual symptoms

Decreased hearing

Neck mass

Cranial III involvement

Cranial V involvement

Cranial VI involvement

Cranial VII involvement
N (\%)

$45(42.1 \%)$

$38(35.5 \%)$

$32(29.9 \%)$

$33(30.8 \%)$

47 (43.9\%)

$90(84.1 \%)$

$3(2.8 \%)$

$1(0.9 \%)$

$6(5.6 \%)$

$5(4.7 \%)$

TABLE 1: Clinical presentations 


\section{Cureus}

TNM Classifications

N (\%)

T classifications

T1

T2

T3

T4

$\mathrm{N}$ classifications

No

N1

N2

N3

M classifications

Mo

M1

Pathology

Squamous cell carcinoma

Undifferentiated carcinoma

Differentiated was not stated
$9(8.4 \%)$

$23(21.5 \%)$

$35(32.7 \%)$

$40(37.4 \%)$

$10(9.3 \%)$

$20(18.7 \%)$

$45(42.1 \%)$

$32(29.9 \%)$

$90(84.1 \%)$

$17(15.9 \%)$

N (\%)

$16(15 \%)$

$82(76.6 \%)$

$9(8.4 \%)$

\section{TABLE 2: TNM classifications}

TNM: Tumor Nodes Metastasis

The overall five-year survival rate was $81.3 \%$ for the whole group (Figure 2) and was $100 \%$ for stage 1 and $83.3 \%$ for stage 2 . The five-year survival rate was $93.9 \%$ and $72.2 \%$ for those with stage 3 and 4 , respectively. Patients with stage 1 and 2 have higher five-year survival but statistically insignificant ( $p$-value $>0.05$ ).

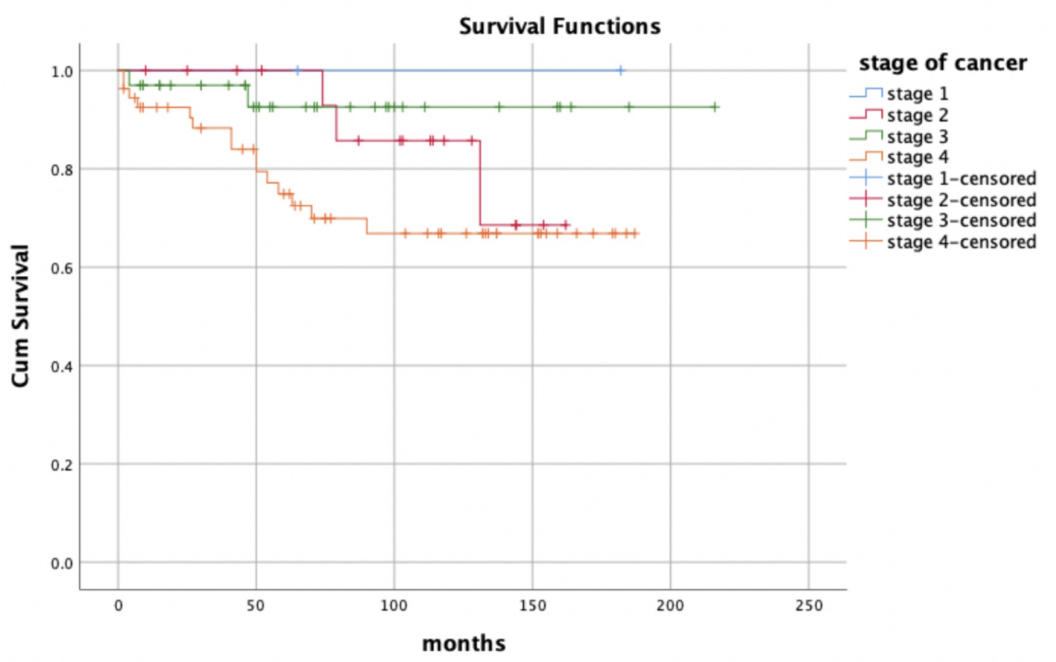

FIGURE 2: Overall five-year survival by stages 
96.3\% patients $(\mathrm{n}=103)$ completed the treatment with radiotherapy and/or chemotherapy, and $20.6 \%$ patients $(n=22)$ underwent any type of surgery. The assessment of quality of life $(\mathrm{Q} o \mathrm{~L})$ of 25 patients, who were in remission, was asked by research coordinator using $\mathrm{H} \& \mathrm{~N}-35$ modules. A calculated score for each patient is shown in Table 3. In the H\&N-35 module, swallowing and social contact ranked the highest scores.

\begin{tabular}{|c|c|c|c|c|c|}
\hline Symptoms & $\mathbf{N}$ & Minimum & Maximum & Mean & Std. Deviation \\
\hline Pain & 25 & 4.00 & 12.00 & 5.4000 & 2.06155 \\
\hline Swallowing & 25 & 4.00 & 12.00 & 7.0400 & 2.57358 \\
\hline Sense Problems & 25 & 2.00 & 6.00 & 3.2400 & 1.12842 \\
\hline Speech Problems & 25 & 3.00 & 9.00 & 4.5600 & 1.93821 \\
\hline Social Eating & 25 & 4.00 & 16.00 & 6.7200 & 3.22128 \\
\hline Social Contact & 25 & 5.00 & 18.00 & 7.3200 & 3.09192 \\
\hline Less Sexuality & 25 & 2.00 & 10.00 & 4.2400 & 2.94788 \\
\hline Teeth & 25 & 1.00 & 4.00 & 1.8800 & 0.88129 \\
\hline Opening Mouth & 25 & 1.00 & 4.00 & 2.0400 & 1.17189 \\
\hline Dry Mouth & 25 & 1.00 & 4.00 & 2.1200 & 1.05357 \\
\hline Sticky Saliva & 25 & 1.00 & 4.00 & 2.2800 & 0.89069 \\
\hline Coughing & 25 & 1.00 & 3.00 & 1.7200 & 0.73711 \\
\hline Feel of Illness & 25 & 1.00 & 4.00 & 1.6400 & 0.86023 \\
\hline Pain Killers & 25 & 6.00 & 7.00 & 6.5200 & 0.50990 \\
\hline Nutritional Supplements & 25 & 6.00 & 7.00 & 6.5200 & 0.50990 \\
\hline Feeding Tube & 25 & 6.00 & 7.00 & 6.9200 & 0.27689 \\
\hline Weight Loss & 25 & 6.00 & 7.00 & 6.8000 & 0.40825 \\
\hline Weight Gain & 25 & 6.00 & 7.00 & 6.2400 & 0.43589 \\
\hline
\end{tabular}

TABLE 3: Calculated scores for QLQ-H\&N-35

\section{Discussion}

Patients presenting with early stage disease had a higher five-year survival rate though not statistically significant, compared to more advanced stage, with stage 1 being $100 \%$. Similarly, a study that was done in China concluded that stage 1 NPC patients had 100\% five-year survival rate [21]. In practice, the commonest presentation in our series was neck nodes, denoting more advanced stage. Furthermore, the incidence rate of our NPC patients was higher in males compared to females with a male-to-female ratio of almost 3:1, similar to the results from the International Agency for Research on Cancer at WHO (WHO-IARC) that is seen in NPC patients in Kuwait [22]. The association between mortality rate and patients' risk factors such as diabetes, hypertension, and smoking was statistically insignificant in our study.

From the quality of life results, the social aspect was the most affected, as patients reported that they had issues with their social life. $72 \%$ of the surveyed patients had difficulty staying in touch with relatives, $64 \%$ reported having problems while eating a meal with others and $36 \%$ stated having speech difficulties. These multiple social difficulties may present in patients with various cancer types, however, we believe that the social aspect is crucial in NPC patients because of the younger age, and higher cure rate.

In addition, only $12 \%$ of the patients in our study reported a feeling of illness, and $16 \%$ reported they need to use any type of painkillers. Also, $16 \%$ reported having mouth dryness, sticky saliva and cough. Though not related to the social aspect, these may suggest better quality of life in the NPC patients day-to-day living considering that they have cancer. In comparison, a study done in Turkey showed swallowing, mouth, teeth problems and weight gain were higher in their population but statistically insignificant [23]. 
A significant number of our patients were excluded due mainly to incomplete clinical data and/or follow-up, making interpretation of the survival data limited. This is in agreement with another study from Saudi Arabia, where differences in survival rates lacked statistical significance due to the low number of patients and a loss of follow-ups [24].

\section{Conclusions}

This study included 15-year retrospective data about nasopharyngeal cancer patients in Princess Norah Center for Cancer, and we were able to identify 107 NPC patients with adequate information. Higher incidence rate was in male patients, and the neck mass was the most common clinical presentation that accounted for $84.1 \%$. In addition, there was no association between mortality rate and patients' co-morbid factors. Also, social contact among our patients was a major issue affecting the quality of life. Future studies must focus on ways to improve the functional outcome of this young patient population.

\section{Additional Information \\ Disclosures}

Human subjects: All authors have confirmed that this study did not involve human participants or tissue. Animal subjects: All authors have confirmed that this study did not involve animal subjects or tissue. Conflicts of interest: In compliance with the ICMJE uniform disclosure form, all authors declare the following: Payment/services info: All authors have declared that no financial support was received from any organization for the submitted work. Financial relationships: All authors have declared that they have no financial relationships at present or within the previous three years with any organizations that might have an interest in the submitted work. Other relationships: All authors have declared that there are no other relationships or activities that could appear to have influenced the submitted work.

\section{Acknowledgements}

Acknowledgment to Reem Asiri and Ziad Bukhari for their efforts.

\section{References}

1. World Health Organization. Cancer. (2017). Accessed: January 31, 2018: https://www.who.int/en/newsroom/fact-sheets/detail/cancer.

2. Global Burden of Disease Cancer Collaboration, Fitzmaurice C, Allen C, et al.: Global, regional, and national cancer incidence, mortality, years of life lost, years lived with disability, and disability-adjusted life-years for 32 cancer groups 1990 to 2015: a systematic analysis for the global burden of disease study. JAMA. 2017, 3:524-548. 10.1001/jamaoncol.2016.5688

3. Devita VT, Hellman S, Rosenberg SA: Principles and Practice of Oncology. Lippincott, Williams \& Wilkims, Philadelphia; 2001.

4. Tsao SW, Huang DP, Lo KW: Nasopharyngeal carcinoma. In: Epstein-Barr Virus. Tselis AC, Jenson H (ed): Taylor \& Francis, New York; 2006. 24. 10.3109/9781420014280

5. Yu M, Yuan J: Epidemiology of nasopharyngeal carcinoma. Semin Cancer Biol. 2002, 12:421-429. 10.1016/S1044579X02000858

6. Bazarbashi S, Al-Eid H, Minguet J: Cancer incidence in Saudi Arabia: 2012 data from the Saudi cancer registry. Asian Pac J Cancer Prev: APJCP. 2017, 18:2437-2444. 10.22034/APJCP.2017.18.9.2437

7. Hui EP, Chan A: Epidemiology, etiology, and diagnosis of nasopharyngeal carcinoma. UpToDate. 2017,

8. Tsui EY, Chan JH, Ramsey RG, et al.: Late temporal lobe necrosis in patients with nasopharyngeal carcinoma: evaluation with combined multi-section diffusion weighted and perfusion weighted MR imaging. Eur J Radiol. 2001, 39:133-138. 10.1016/S0720-048X(01)00328-X

9. Lee AW, Ng WT, Chan YH, Sze H, Chan C, Lam TH: The battle against nasopharyngeal cancer . Radiother Oncol. 2012, 104:272-278. 10.1016/j.radonc.2012.08.001

10. Chua ML, Wee JT, Hui EP, Chan AT: Nasopharyngeal carcinoma. Lancet. 2016, 387:1012-1024. 10.1016/S0140-6736(15)00055-0

11. Pathmanathan R, Prasad U, Sadler R, Flynn K, Raab-Traub N: Clonal proliferations of cells infected with Epstein-Barr virus in preinvasive lesions related to nasopharyngeal carcinoma. N Engl J Med. 1995, 333:693698. 10.1056/NEJM199509143331103

12. Greene MH, Fraumeni JF Jr, Hoover R: Nasopharyngeal cancer among young people in the United States: racial variations by cell type. J Natl Cancer Inst. 1977, 58:1267-1270. 10.1093/jnci/58.5.1267

13. Berry MP, Smith CR, Brown TC, Jenkin RD, Rider WD: Nasopharyngeal carcinoma in the young. Int J Radiat Oncol Biol Phys. 1980, 6:415-421. 10.1016/0360-3016(80)90054-1

14. Sham JS, Poon YF, Wei WI, Choy D: Nasopharyngeal carcinoma in young patients. Cancer. 1990, 65:26062610. 10.1002/1097-0142(19900601)65:11<2606::aid-cncr2820651135>3.0.c0;2-u

15. Lucente FE, Har-El G: Essentials of Otolaryngology. Wolters Kluwer, 2004.

16. Asherson N: Trotter's syndrome and associated lesions. J Laryngol Otol. 1951, 65:349-366. 10.1017/S002221510001015X

17. Suárez C, Rodrigo JP, Rinaldo A, Langendijk JA, Shaha AR, Ferlito A: Current treatment options for recurrent nasopharyngeal cancer. Eur Arch Otorhinolaryngol. 2010, 267:1811-1824. 10.1007/s00405-0101385-X

18. List MA, Stracks J: Evaluation of quality of life in patients definitively treated for squamous carcinoma of the head and neck. Curr Opin Oncol. 2000, 12:215-220.

19. Jang-Chun L, Jing-Min H, Yee-Min J, et al.: Comparisons of quality of life for patients with nasopharyngeal 


\section{Cureus}

carcinoma after treatment with different RT technologies. Acta Otorhinolaryngol Ital. 2014, 34:241-246.

20. Greene FL, Page DL, Fleming ID, et al.: AJCC Cancer Staging Manual. Springer Science and Business Media, Berlin, Germany; 2013.

21. Wu L, Liu Y, Jiang N, et al.: Ten-year survival outcomes for patients with nasopharyngeal carcinoma receiving intensity-modulated radiotherapy: an analysis of 614 patients from a single center. Oral Oncol. 2017, 69:26-32.10.1016/j.oraloncology.2017.03.015

22. Whelan SL, Parkin DM, Masuyer E: Pattern of Cancer in Five Continents. IARC Scientific Publication, Lyon; 1990.

23. Cengiz M, Özyar E, Esassolak M, et al.: Assessment of quality of life of nasopharyngeal carcinoma patients with EORTC QLQ-C30 and H\&N-35 modules. Int J Radiat Oncol Biol Phys. 2005, 63:1347-1353. 10.1016/j.ijrobp.2005.05.057

24. Al-Wassia R, Abusanad A, Awad N, Marzouki H, Alkhayyat S, Al-Khatib T, Constantinescu C: Outcomes of Saudi Arabian patients with nasopharyngeal cancer treated with primarily neoadjuvant chemotherapy followed by concurrent chemoradiotherapy. J Global Oncol. 2016, 2:123-128. 10.1200/jgo.2015.001743 